\title{
MicroRNA-409-3p suppresses cell proliferation and cell cycle progression by targeting cyclin D2 in papillary thyroid carcinoma
}

\author{
ZHIJUN ZHAO, FEI YANG, YAN LIU, KAI FU and SHANGHUA JING \\ Department of Head and Neck Surgery, The Fourth Hospital of Hebei Medical University, \\ Shijiazhuang, Hebei 050000, P.R. China
}

Received January 11, 2017; Accepted December 6, 2017

DOI: $10.3892 /$ ol.2018.9246

\begin{abstract}
MicroRNAs (miRs) function as tumor suppressors or oncogenes in tumor development and progression. The present study reported that miR-409-3p was significantly downregulated in human papillary thyroid carcinoma (PTC) tissues and cell lines. Overexpression of miR-409-3p suppressed cell proliferation and induced cell cycle $G_{0} / G_{1}$ phase arrest in PTC cells. Further study revealed that the transcriptional regulator cyclin D2 was a target of miR-409-3p, as miR-409-3p bound directly to its 3'-untranslated region and the miR-409-3p mimic reduced the protein expression levels of cyclin D2. In addition, restoration of cyclin D2 expression reversed the inhibitive effect of miR-409-3p on PTC cells. To the best of our knowledge, these findings demonstrate for the first time that miR-409-3p functions as a tumor suppressor in PTC and could serve as an efficient agent for therapy of PTC.
\end{abstract}

\section{Introduction}

Thyroid cancer is the most common endocrine malignancy in the world, with a rapid increasing incidence (1); it has been classified into several subtypes, including papillary thyroid cancer (PTC), follicular thyroid cancer, medullary thyroid cancer and anaplastic thyroid cancer (2). PTC is the most common type and is derived from follicular thyroid cells and accounts for $\sim 80 \%$ of thyroid cancer cases (3). Currently, the majority of PTC patients that undergo surgical resection in combination with radioiodine and levothyroxine have a good prognosis; however, recurrence is observed in $\sim 30 \%$ of patients (4). Therefore, understanding the molecular mechanism underlying the development and progression of PTC is necessary to elucidate more effective therapeutic strategies.

Correspondence to: Mr. Shanghua Jing, Department of Head and Neck Surgery, The Fourth Hospital of Hebei Medical University, 12 Health Road, Shijiazhuang, Hebei 050000, P.R. China

E-mail: jingshua1962@126.com

Key words: papillary thyroid carcinoma, microRNA-409-3p, cell proliferation, cyclin D2
MicroRNAs (miRNAs/miRs) are a group of small endogenous non-coding RNAs with a length of 19-22 nucleotides, which are derived from double-stranded RNA (5). miRs are capable of regulating many biological processes, including cell differentiation, apoptosis and development through the complimentary binding of the 3'-untranslated region (3'-UTR) of target genes, resulting in the degradation or silencing of the target mRNA $(6,7)$. Evidence indicates that the alteration of miRNAs was frequently associated with cancer progression, the miRNAs acting as tumor suppressors or oncogenes depending on the cellular function of their targets $(8,9)$. A number of miRNAs have been identified to contribute to PTC development and progression, including miR-199a-3p (10), miR-101 (11) and miR-34a (12). Previously, miR-409-3p has been reported to be a potential tumor suppressor in variety of cancer types, including colorectal (13), prostate (14) and breast (15) cancer. However, to the best of our knowledge, no evidence of miR-409-3p function in PTC has been documented.

In this study, the expression of miR-409-3p in human PTC tissues and cells was determined. In vitro experiments were performed to investigate the function role of miR-409-3p in PTC cells, as well as the underlying mechanisms. The findings of the present study suggest that miR-409-3p may be a potential target for PTC treatment and serve an important role in development and progression of PTC.

\section{Materials and methods}

Clinical tissue specimens and cell lines. Samples of 20 pairs of primary PTC tissues and adjacent non-tumor tissues $(\geq 3 \mathrm{~cm}$ away from the primary site) were obtained from patients with thyroid cancer, who had undergone surgical resection at the Fourth Hospital of Hebei Medical University (Hebei, China). Specimens were collected, immediately snap-frozen in liquid nitrogen and stored at $-80^{\circ} \mathrm{C}$ until RNA was extracted. In the present study, each patient voluntarily signed written informed consent and the collection and use of patient samples was approved by the Ethics Committee of the Fourth Hospital of Hebei Medical University.

Human PTC B-CPAP, TPC-1 and GLAG-66 cell lines and the human thyroid epithelial Nthy-ori3-1 cell line were purchased from the Type Culture Collection of the Chinese Academy of Sciences (Shanghai, China). All cell lines were cultured in RPMI-1640 medium (Invitrogen; Thermo Fisher Scientific, 
Inc., Waltham, MA, USA) supplemented with $10 \%$ fetal bovine serum (Sigma-Aldrich, Merck, KGaA, Darmstadt, Germany) in a humidified incubator of $5 \% \mathrm{CO}_{2}$ at $37^{\circ} \mathrm{C}$.

Cell transfection. The synthesized oligos for miR-409-3p mimics (5'-GAAUGUUGCUCGGUGAACCCCU-3') or negative control (NC: 5'-ACTACTGAGTGACAGTAGA-3') were purchased from Sangon Biotech Co., Ltd. (Shanghai, China). For cyclin D2 overexpression, the coding sequence of human cyclin D2 was cloned into a pcDNA3.1 vector (performed by Thermo Fisher Scientific, Inc.). The empty pcDNA3.1 was used as a negative control. Next, TPC-1 and GLAG-66 cells were seeded into 24 -well plates at a density of $4 \times 10^{5}$ cells per well and allowed to attach prior to transfection to ensure $60-70 \%$ cell confluence and transfected with the aforementioned vectors using Lipofectamine 2000 (Invitrogen; Thermo Fisher Scientific, Inc.). The miRNA mimics were used at a final concentration of $50 \mathrm{nM}$. Cell samples were collected at $48 \mathrm{~h}$ after transfection for further analysis.

Reverse transcription-quantitative polymerase chain reaction (RT-qPCR). RNA was isolated from tissues and PTC cells with TRIzol ${ }^{\circledR}$ reagent (Invitrogen; Thermo Fisher Scientific, Inc.) and RNA templates were reverse transcribed into cDNA using a One Step PrimeScript cDNA Synthesis kit (Takara Bio, Inc., Otsu, Japan) according to the manufacturer's protocol. miR-409-3p and CCND2 expression levels were quantified using a ABI 7500 FAST real-time PCR system (Applied Biosystems; Thermo Fisher Scientific, Inc.) using SYBR Green PCR Master Mix (Takara Bio, Inc.). The thermocycling conditions of the PCR reaction were as follows: Initial denaturation for $1 \mathrm{~min}$ at $95^{\circ} \mathrm{C}$, denaturation for $5 \mathrm{sec}$ at $95^{\circ} \mathrm{C}$, and annealing for 40 cycles at $60^{\circ} \mathrm{C}$. The expression of U6 and GAPDH were used to normalize miR-409-3p and CCND2 expression levels in each group, respectively, using the $2^{-\Delta \Delta C q}$ method (16). The primer sequences were as follows: miR-409-3p forward, GAA TGTTGCTCGGTGAACCCCT and reverse, GAAUGUUGC UCGGUGAACCCCU; CCND2 forward, TGCAACCGA CGATTCTTCTACTCAA and reverse, CAAGCAGTGATG TATCTGATAAACAAGG; U6 forward, CTCGCTTCGGCA GCACA and reverse, AACGCTTCACGAATTTGCGT; and GAPDH forward, AGAGGCAGGGATGATGTTCTG and reverse, GACTCATGACCACAGTCCATGC.

Cell proliferation assay. For cell proliferation assay, approximately 4x10 3 TPC-1 and GLAG-66 cells following transfection were seeded in a 96-well plate. After removing the medium, Cell Counting Kit-8 solution (CCK-8; Dojindo Molecular Technologies, Inc., Kumamoto, Japan) was added to cells and incubated at $37^{\circ} \mathrm{C}$ for an additional $2 \mathrm{~h}$. The absorbance of the solution at a wavelength of $450 \mathrm{~nm}$ was measured with a MRX II absorbance reader (Dynex Technologies, Worthing, UK).

Colony formation assay. After $48 \mathrm{~h}$ transfection, TPC-1 and GLAG-66 cells at a density of 500 cells per well were seeded into 6-well plates and incubated for 7 days at $37^{\circ} \mathrm{C}$. Subsequently, the cells were washed twice with PBS, fixed in $70 \%$ ethanol and stained with $1 \%$ crystal violet solution for $30 \mathrm{~min}$ at room temperature. Colonies containing $>50$ cells were photographed and counted using a light microscope (x200 magnification).

Cell cycle analysis. Cell cycle distribution was determined using flow cytometry with propidium iodide (PI) staining (Sigma-Aldrich, Merck KGaA Darmstadt Germany). Briefly, cells were plated in $6-\mathrm{cm}$ dishes at a density of $1.0 \times 10^{5}$ cells per dish after transfection for $48 \mathrm{~h}$. Next, cells were washed with PBS and fixed in $70 \%$ ethanol overnight at $4^{\circ} \mathrm{C}$. The following day, the cells were stained with a PI/RNase Staining Solution (Beyotime Institute of Biotechnology) at $4^{\circ} \mathrm{C}$ for $25 \mathrm{~min}$. Cellular DNA was analyzed on fluorescence-activated cells sorting (FACS) Calibur flow cytometer (Gallios, Beckman Coulter, Inc., Brea, CA, USA).

Dual-luciferase reporter assays. The potential target genes of miR-409-3p were predicted using TargetScan database (http://www.targetscan.org/vert_71/). The 3'-UTR of CCND2 containing predicted miR-409-3p binding sites (5'-UGACAU UCCCAUCACAACAUUC-3') was amplified from human cDNA and cloned into the psiCHECK-2 dual-luciferase expression vector by Promega Corporation (Madison, WI, USA). The mutation of the predicted 3'-UTR was obtained using PCR site-directed mutagenesis by Promega Corporation (Madison, WI, USA). 293T cells were seeded into 48-well plates, then co-transfected with miR-409-3p mimics or NC and luciferase reporter plasmids $(50 \mathrm{ng}$ ) containing the psiCHECK-cyclin D2-3'-UTR wild type or mutant (5'-UGACAUUCCCAUCAG TTGTAAC-3') using Lipofectamine 2000 (Thermo Fisher Scientific, Inc.) for $24 \mathrm{~h}$ at $37^{\circ} \mathrm{C}$. Firefly and Renilla luciferase activities were measured at $48 \mathrm{~h}$ after transfection using a dual-luciferase reporter assay system (Promega Corporation). As per the previous experiment an empty vector control was usedto validate that there was no-specific effects from vector integration into the hosts genome during transfection. Results were presented as the ratio of Renilla luciferase activity to firefly luciferase activity.

Western blot analysis. At $48 \mathrm{~h}$ after transfection, cells were harvested, lysed using RIPA lysis buffer (Beyotime Institute of Biotechnology) and the protein concentration in samples was quantified with the Bicinchoninic Acid Protein Assay kit (Beyotime Institute of Biotechnology). Equivalent quantities (30 $\mu \mathrm{g}$ per lane) of protein were separated by $10 \%$ SDS-PAGE and transferred to polyvinylidene difluoride membranes (Bio-Rad Laboratories, Inc., Hercules, CA, USA). Membranes were blocked for $1 \mathrm{~h}$ with $5 \%$ non-fat milk at room temperature and then incubated overnight at $4{ }^{\circ} \mathrm{C}$ with primary antibodies, including anti-cyclin D2 (1:1,000, ab81359, Abcam, Cambridge, UK) and anti-GAPDH (1:10,000, 10494-1-AP, ProteinTech Group, Inc., Chicago, IL, USA). Next, the membranes were washed three times in TBST containing $0.1 \%$ Tween-20 and incubated with the corresponding horseradish peroxidase-conjugated goat anti-rabbit (1:5,000; sc-2054, Santa Cruz Biotechnology, Inc., Dallas, TX, USA) at room temperature for $1 \mathrm{~h}$. The blots were visualized using an enhanced chemiluminescence system (BeyoECLPlus; Beyotime Institute of Biotechnology). Band density was measured by Image-Pro Plus version 6.0 software (Media Cybernetics, Inc., Rockville, MD, USA). 
A

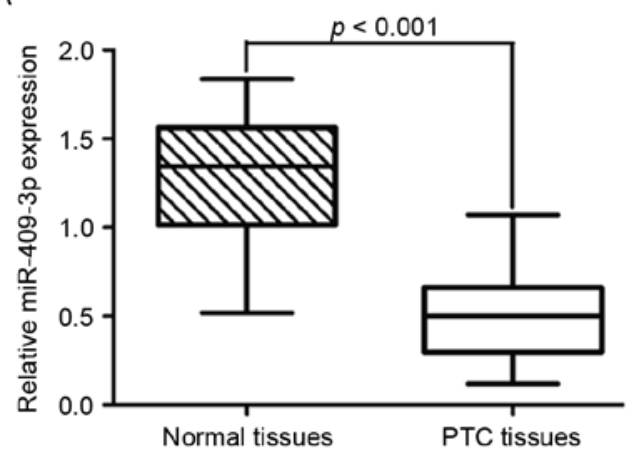

B

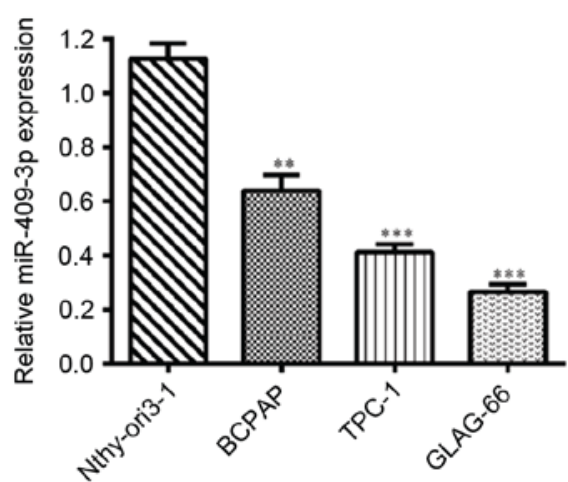

Figure 1. Downregulation of miR-409-3p expression in PTC tissues and cell lines compared with the corresponding controls. Reverse transcription-quantitative polymerase chain reaction was used to analyze the relative expression levels of miR-409-3p in (A) 20 surgically resected specimens of PTC tissues and matched non-tumor tissues, as well as in (B) three PTC cell lines (B-CPAP, TPC-1 and GLAG-66) and a normal human thyroid epithelial cell line (Nthy-ori3-1). Data are shown as the mean and standard deviation of expression levels relative to U6 expression levels. ${ }^{* *} \mathrm{P}<0.01,{ }^{* * * *} \mathrm{P}<0.001$ vs. Nthy-ori3-1. PTC, papillary thyroid carcinoma; miR-409-3p, microRNA-409-3p.

Statistical analysis. All experiments were repeated in triplicate and the results were expressed as the mean \pm standard deviation. All analyses were performed using GraphPad Prism 5 software (GraphPad Software, Inc., La Jolla, CA, USA). A two-tailed Student's t-test was applied for comparisons between the two groups. Multiple comparisons were performed using a one-way analysis of variance followed by Tukey's post hoc test. $\mathrm{P}<0.05$ was considered to indicate a statistically significant difference.

\section{Results}

Expression of miR-409-3p is downregulated in PTC tissues and cell lines. The expression levels of miR-409-3p were first determined by RT-qPCR in 20 pairs of human PTC tissues and adjacent normal tissues. As shown in Fig. 1A, the expression level of miR-409-3p was significantly lower in tumor tissues compared with matched non-tumor tissues $(\mathrm{P}<0.001)$. Also, the expression levels of miR-409-3p were measured in three PTC cell lines (B-CPAP, TPC-1 and GLAG-66) and a normal human thyroid epithelial cell line (Nthy-ori3-1). miR-409-3p was significantly downregulated in all PTC cell lines compared with Nthy-ori3-1 (Fig. 1B; $\mathrm{P}<0.001$ ). Thus, it was concluded that a decrease in miR-409-3p expression may serve a role in PTC development.

Overexpression of miR-409-3p induces cell growth inhibition and $G_{0} / G_{1}$ phase arrest in PTC cells. The results demonstrated that TPC-1 and GLAG-66 cells presented lower expression of miR-409-3p. Therefore, synthetic miR-409-3p mimics and NC mimics were separately transfected into TPC-1 and GLAG-66 cells to investigate the biological function of miR-409-3p in PTC. RT-qPCR assays revealed that miR-409-3p expression was significantly upregulated following transfection with miR-409-3p mimics, compared with NC control cells in TPC and GLAG-66 cells ( $\mathrm{P}<0.001$; Fig. 2A). The CCK-8 assay demonstrated that transfection with miR-409-3p mimics significantly inhibited cell proliferation in TPC-1 and GLAG-66 cells $(\mathrm{P}<0.05$; Fig. $2 \mathrm{~B})$. The colony formation assay indicated that the ability of colony formation in TPC-1 and GLAG-66 cells transient transfected with miR-409-3p mimics was impaired compared with that in NC-transfected cells $(\mathrm{P}<0.05$; Fig. 2C). Flow cytometric analysis revealed that overexpression of miR-409-3p led to $G_{0} / G_{1}$ phase arrest by elevating the percentage of cells at $\mathrm{G}_{0} / \mathrm{G}_{1}$ phase in TPC-1 and GLAG-66 cells, but decreasing the cell numbers at $\mathrm{S}$ and $\mathrm{G}_{2} / \mathrm{M}$ phase in TPC cells and cells at $\mathrm{G}_{2} / \mathrm{M}$ phase in GLAG-66 cells $(\mathrm{P}<0.05$; Fig. 2D).

miR-409-3p downregulates cyclin D2 expression by targeting its 3'UTR. On the basis of the aforementioned results, a possible role for miR-409-3p in repressing $G_{0} / G_{1}$-associated genes was hypothesized. As shown in Fig. 3A, CCND2 exhibited miR-409-3p-binding sequences in its 3'-UTR, which was identified as a putative target of miR-409-3p by miRNA target gene prediction. To confirm the direct targeting of CCND2 by miR-409-3p, a dual-luciferase reporter assay was performed by integrating sequences of the CCND2 3'UTR containing the binding sites for miR-409-3p or corresponding mutated sequences into $293 \mathrm{~T}$ cells. Luciferase assays revealed that miR-409-3p mimics significantly decreased the relative activity of the luciferase reporter containing the wild-type 3'-UTR of CCND2 mRNA $(\mathrm{P}<0.05)$, but did not affect the luciferase activity of the mutant CCND2 3'-UTR (Fig. 3B). Next, the effect of miR-409-3p on the expression of CCND2 was measured at the mRNA and protein levels in PTC cells. As shown in Fig. 3C, transfection with miR-409-3p mimics downregulated the mRNA and protein expression levels of CCND2 and cyclin D2, respectively, compared with cells transfected with $\mathrm{NC}$ controls $(\mathrm{P}<0.05)$. These results indicated that CCND2 may be a direct target of miR-409-3p in PTC.

Enhanced expression of CCND2 partially rescues miR-409-3p-induced cell growth inhibition and $G_{0} / G_{1}$ phase arrest in TPC cells. To determine whether CCND2 mediated the inhibitory effect of miR-409-3p on cell proliferation and cell cycle progression, rescue experiments were performed by transfecting pcDNA/CCND2 into PTC cells with higher miR-409-3p expression levels and cell proliferation and cell cycle were then examined. The results revealed that 
A

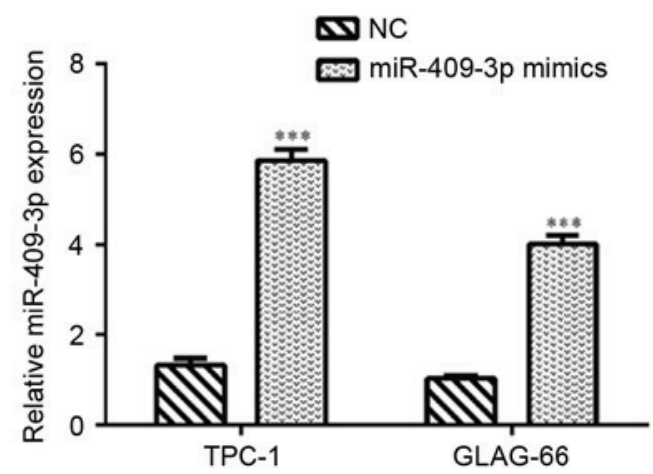

C

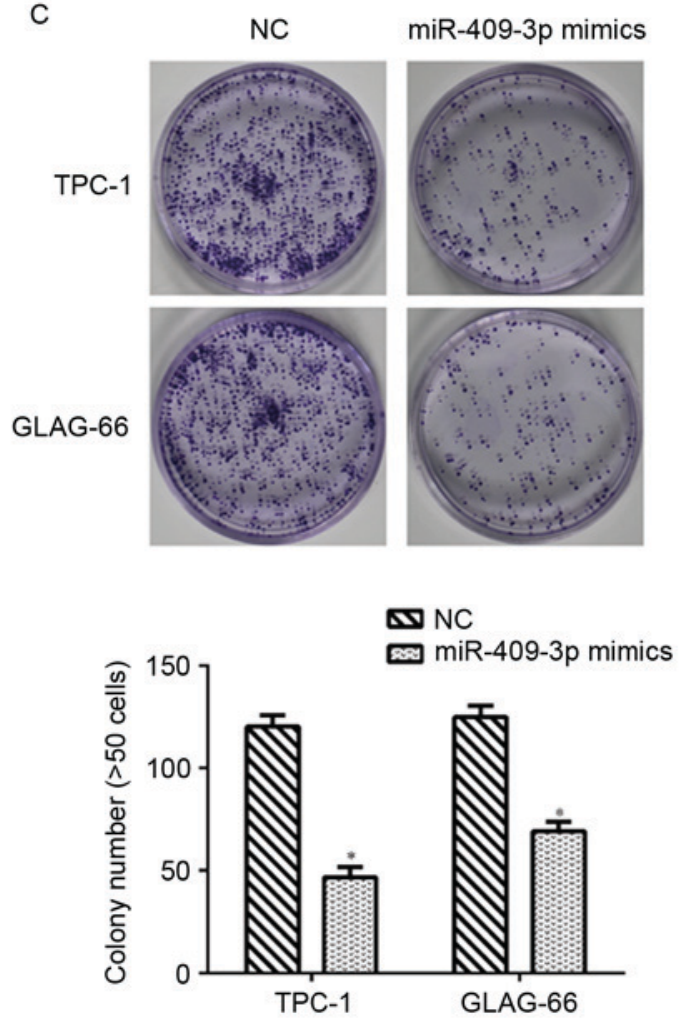

B

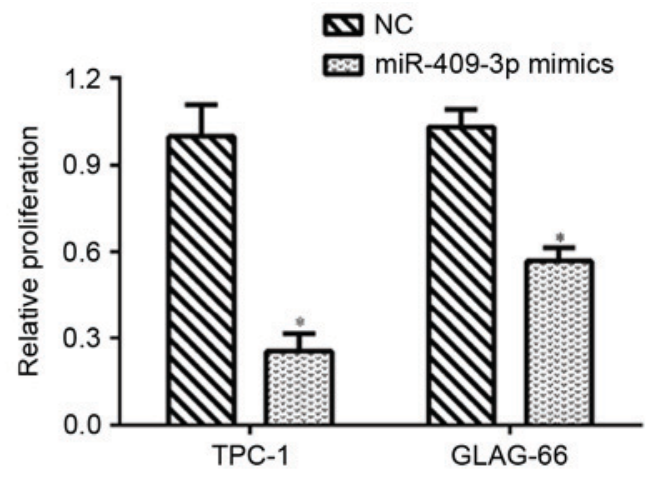

D
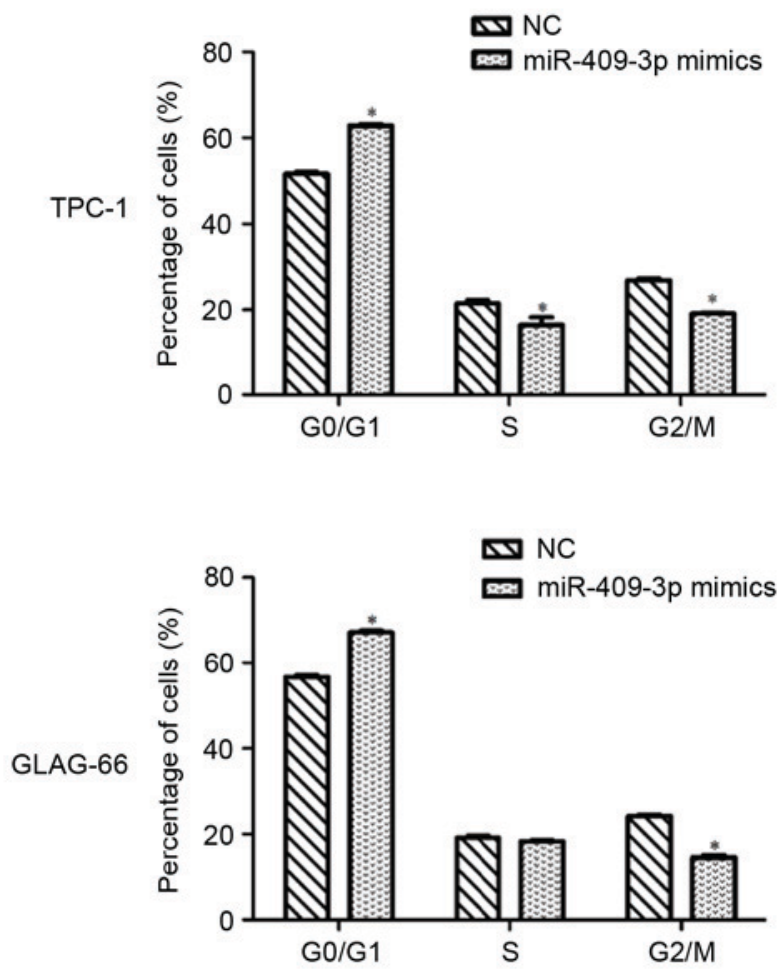

Figure 2. Overexpression of miR-409-3p inhibits PTC cell proliferation by cell cycle arrest at $\mathrm{G}_{0} / \mathrm{G}_{1}$ phase. (A) Reverse transcription-quantitative polymerase chain reaction detection of miR-409-3p expression in TPC-1 and GLAG-66 cells stably transfected with miR-409-3p mimics or NC. U6 small nuclear RNA was used as an internal control. (B) Effect of miR-409-3p overexpression on PTC cell viability using a Cell Counting Kit-8 assay. (C) Representative micrographs of cell colony formation assay (top) and quantification (bottom) from three independent experiments. (D) Flow cytometry cell cycle analysis revealed the effect of miR-409-3p mimic transfection on the percentage of cells in $\mathrm{G}_{0} / \mathrm{G}_{1}, \mathrm{~S}$ and $\mathrm{G}_{2} / \mathrm{M}$ phase in TPC-1 and GLAG-66 cells. Values represent the mean from three separate experiments and error bars represent the standard deviation. ${ }^{*} \mathrm{P}<0.05$ and ${ }^{* * *} \mathrm{P}<0.001$ vs. NC. PTC, papillary thyroid carcinoma; miR-409-3p, microRNA-409-3p; NC, negative control.

overexpression of CCND2 partially rescued the impaired cell proliferation induced by miR-409-3p mimics in TPC-1 and GLAG-66 cells $(\mathrm{P}<0.05$; Fig. 4A). It was also found that restoration of $C C N D 2$ reversed the cell cycle $\mathrm{G}_{0} / \mathrm{G}_{1}$ phase arrest imposed by miR-409-3p mimics in TPC-1 $(\mathrm{P}<0.05$; Fig. 4B) and GLAG-66 cells ( $\mathrm{P}<0.05$; Fig. 4C). These data indicated that $C C N D 2$ may mediate the suppressive functions of miR-409-3p in PTC cells.

\section{Discussion}

In recent years, many miRNA signatures have been well characterized for PTC (17); however, but further investigation on the roles of dysregulated miRs in PTC development and progression is necessary for seeking more effective therapeutic strategies. In the present study, it was observed that miR-409-3p was frequently downregulated in PTC samples specimens and cell lines, consistent with its reduced expression in lung (18) and bladder cancer (19). Functional analysis revealed that overexpression of miR-409-3p caused a reduction in proliferation rate, which was accompanied by $\mathrm{G}_{0} / \mathrm{G}_{1}$ phase arrest of the cell cycle, indicating its suppressive role in PTC cells.

Evidence of miR-409-3p as a tumor growth inhibitor has been well documented in several types of cancer. For example, miR-409-3p inhibited growth and induced apoptosis in gastric cancer (20). In breast cancer, miR-409-3p has been demonstrated to suppress cell growth by targeting protein 
A

\begin{tabular}{ll}
\hline CCND2 wt & 5'-UGACAUUCCCAUCACAACAUUC-3' \\
has-miR-409-3p & 3'-UCCCCAAGUGGCUCGUUGUAAG-5' \\
CCND2 mut & 5'-UGACAUUCCCAUCAGTTGTAAC-3' \\
\hline
\end{tabular}

B

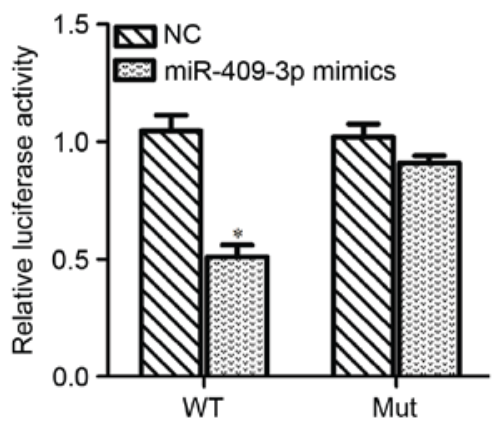

C

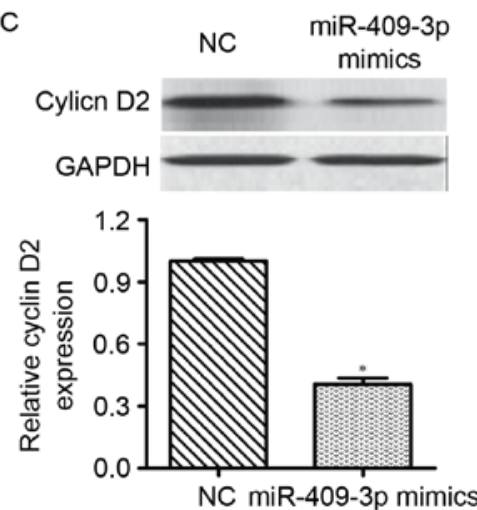

Figure 3. miR-409-3p directly binds to the 3'-UTR of CCND2. (A) Predicting binding sites and the corresponding mutated sequences within the CCND2 3'UTR for miR-409-3p were presented. (B) 293T cells were co-transfected with either miR-409-3p mimics or NC and luciferase reporter psiCHECK vector comprising Wt or Mut 3'UTR of CCND2. The relative firefly luciferase activity normalized with Renilla luciferase was measured $48 \mathrm{~h}$ after transfection. The data shown represents the mean \pm standard deviation of three independent experiments. (C) The relative CCND2 mRNA and protein levels were detected by reverse transcription-quantitative polymerase chain reaction and western blot analysis, respectively, in PTC cells stably transfected with miR-409-3p mimics or NC. GAPDH was used as an internal control. "P<0.05 vs. NC. 3'UTR, 3' untranslated region; NC, negative control; CCND2, cyclin D2; miR-409-3p, microRNA-409-3p; wt, wild type; mut, mutant; PTC, papillary thyroid carcinoma.
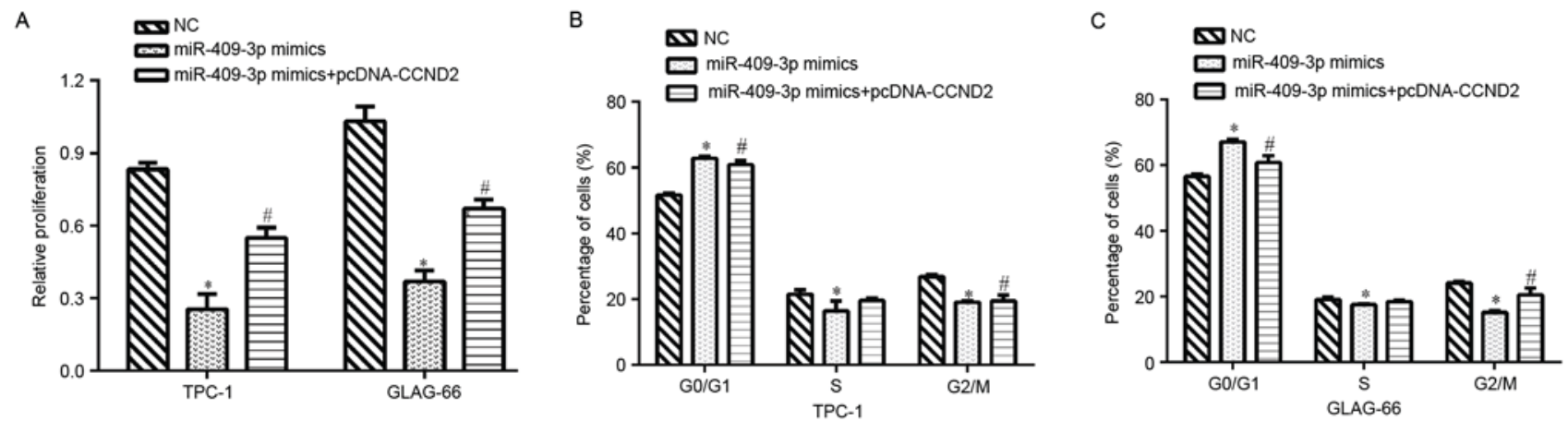

Figure 4. Overexpression of $C C N D 2$ partially rescues the inhibitory effects of miR-409-3p on cell proliferation and cell cycle progression. TPC-1 and GLAG-66 cells were transfected with mimic NC or miR-409-3p mimics together with a plasmid expressing CCND2 or an empty vector. (A) CCK-8 assays were performed to determine the proliferation rate of TPC-1 and GLAG-66 cells after $48 \mathrm{~h}$ transfection. Cell cycle analysis was performed $48 \mathrm{~h}$ after transfection in (B) TPC-1 and (C) GLAG-66 cells. Values represent the mean from three separate experiments; error bars represent the standard deviation. "P $<0.05$ vs. NC and ${ }^{\#} \mathrm{P}<0.05$ vs. miR-409-3p mimics. CCND2, gene encoding cyclin D2; miR-409-3p, microRNA-409-3p; NC, negative control.

kinase B (15). Consistent with the role of miR-409-3p in PTC cell proliferation, the present study identified CCND2 as a target gene of miR-409-3p. Further analysis demonstrated that the enforced expression of miR-409-3p significantly decreased cyclin D2 protein expression levels, indicating that miR-409-3p may be able to negatively regulate the expression of $C C N D 2$. The identification of $C C N D 2$ may account for one of the underlying mechanisms by which miR-409-3p is involved in PTC cell proliferation.

D-type cyclins are reported to be closely associated with cell cycle progression (21), of which CCND2 is a key player in the cell cycle from the $\mathrm{G}_{0} / \mathrm{G}_{1}$ phase to $\mathrm{S}$ phase (22). The $C C N D 2$ oncogene has been well noted in human thyroid cancer, which was downregulated by miR-1 (23). Similarly, upregulation of CCND2 has been documented in breast cancer (24) and prostate cancer (25). To confirm the role of CCND2 in PTC further, the present study also demonstrated that when miR-409-3p-expressing cells resumed CCND2 expression, the proliferation deficiencies and $G_{0} / G_{1}$ phase arrest were partly reversed. Therefore, it was concluded that miR-409-3p may inhibit cell proliferation and cell cycle progression in PTC by downregulating, at least partially, the protein expression of cyclin D2.

In conclusion, the results of the present study indicated that miR-409-3p is frequently downregulated in PTC and may negatively regulate PTC cell proliferation and cell cycle progression by downregulating its target gene $C C N D 2$. The data may help to further elucidate the molecular mechanisms 
underlying PTC progression and provide a strategy for targeting the miR-409-3p/CCND2 interaction as a novel prevention and treatment of PTC.

\section{Acknowledgements}

Not applicable.

\section{Funding}

No funding received.

\section{Availability of data and materials}

All data generated or analyzed during the current study are included in this published article.

\section{Authors' contributions}

ZZ, FY and YL performed the experiments. KF participated in the interpretation of data. SJ is major contributor and participated in the design of study. All authors revised the manuscript, read and approved the final manuscript.

\section{Ethics approval and consent to participate}

Each patient voluntarily signed written informed consent and the collection and the current study was approved by the Fourth Hospital of Hebei Medical University.

\section{Patient consent for publication}

The written informed consent for publication was signed by all patients in advance and they gave their consent for the publication of any associated data and images.

\section{Competing interests}

The authors declare that they have no competing interests.

\section{References}

1. Siegel RL, Miller KD and Jemal A: Cancer statistics, 2015. CA Cancer J Clin 65: 5-29, 2015.

2. Carneiro RM, Carneiro BA, Agulnik M, Kopp PA and Giles FJ: Targeted therapies in advanced differentiated thyroid cancer. Cancer Treat Rev 41: 690-698, 2015.

3. Lloyd RV, Buehler D and Khanafshar E: Papillary thyroid carcinoma variants. Head Neck Pathol 5: 51-56, 2011

4. Shi X, Liu R, Basolo F, Giannini R, Shen X, Teng D, Guan H, Shan Z, Teng W, Musholt TJ, et al: Differential clinicopathological risk and prognosis of major papillary thyroid cancer variants. J Clin Endocrinol Metab 101: 264-274, 2016.

5. Macfarlane LA and Murphy PR: MicroRNA: Biogenesis, function and role in cancer. Curr Genomics 11: 537-561, 2010.
6. Guo H, Ingolia NT, Weissman JS and Bartel DP: Mammalian microRNAs predominantly act to decrease target mRNA levels. Nature 466: 835-840, 2010.

7. Ebert MS and Sharp PA: Roles for microRNAs in conferring robustness to biological processes. Cell 149: 515-524, 2012.

8. Srivastava A, Goldberger H, Dimtchev A, Ramalinga M, Chijioke J, Marian C, Oermann EK, Uhm S, Kim JS, Chen LN, et al: MicroRNA profiling in prostate cancer-the diagnostic potential of urinary miR-205 and miR-214. PLoS One 8: e76994, 2013.

9. Chen CZ: MicroRNAs as oncogenes and tumor suppressors. N Engl J Med 353: 1768-1771, 2005.

10. Minna E, Romeo P, De Cecco L, Dugo M, Cassinelli G, Pilotti S, Degl'Innocenti D, Lanzi C, Casalini P, Pierotti MA, et al: miR-199a-3p displays tumor suppressor functions in papillary thyroid carcinoma. Oncotarget 5: 2513-2528, 2014.

11. Lin X, Guan H, Li H, Liu L, Liu J, Wei G, Huang Z, Liao Z and $\mathrm{Li}$ Y: miR-101 inhibits cell proliferation by targeting Rac1 in papillary thyroid carcinoma. Biomed Rep 2: 122-126, 2014.

12. Ma Y, Qin H and Cui Y: miR-34a targets GAS1 to promote cell proliferation and inhibit apoptosis in papillary thyroid carcinoma via PI3K/Akt/Bad pathway. Biochem Biophys Res Commun 441: 958-963, 2013

13. BaiR, Weng C, Dong H, Li S, Chen G and Xu Z: MicroRNA-409-3p suppresses colorectal cancer invasion and metastasis partly by targeting GAB1 expression. Int J Cancer 137: 2310-2322, 2015.

14. Josson S, Gururajan M, Hu P, Shao C, Chu GY, Zhau HE, Liu C, Lao K, Lu CL, Lu YT, et al: miR-409-3p/-5p promotes tumorigenesis, epithelial-to-mesenchymal transition, and bone metastasis of human prostate cancer. Clin Cancer Res 20: 4636-4646, 2014

15. Zhang G, Liu Z, Xu H and Yang Q: miR-409-3p suppresses breast cancer cell growth and invasion by targeting Akt1. Biochem Biophys Res Commun 469: 189-195, 2016.

16. Livak KJ and Schmittgen TD: Analysis of relative gene expression data using real-time quantitative PCR and the 2(-Delta Delta C(T)) method. Methods 25: 402-408, 2001.

17. Zhu G, Xie L and Miller D: Expression of MicroRNAs in thyroid carcinoma. Methods Mol Biol 1617: 261-280, 2017.

18. Wan L, Zhu L, Xu J, Lu B, Yang Y, Liu F and Wang Z: MicroRNA-409-3p functions as a tumor suppressor in human lung adenocarcinoma by targeting c-Met. Cell Physiol Biochem 34: 1273-1290, 2014.

19. Xu X, Chen H, Lin Y, Hu Z, Mao Y, Wu J, Xu X, Zhu Y, Li S, Zheng $X$ and Xie L: MicroRNA-409-3p inhibits migration and invasion of bladder cancer cells via targeting c-Met. Mol Cells 36: 62-68, 2013.

20. Li C, Nie H, Wang M, Su L, Li J, Yu B, Wei M, Ju J, Yu Y, Yan M, et al: MicroRNA-409-3p regulates cell proliferation and apoptosis by targeting PHF10 in gastric cancer. Cancer Lett 320: 189-197, 2012.

21. Sherr CJ and Roberts JM: Living with or without cyclins and cyclin-dependent kinases. Genes Dev 18: 2699-2711, 2004.

22. Ando K, Ajchenbaum-Cymbalista F and Griffin JD: Regulation of G1/S transition by cyclins D2 and D3 in hematopoietic cells. Proc Natl Acad Sci USA 90: 9571-9575, 1993.

23. Leone V, D'Angelo D, Rubio I, de Freitas PM, Federico A, Colamaio M, Pallante P, Medeiros-Neto G and Fusco A: MiR-1 is a tumor suppressor in thyroid carcinogenesis targeting CCND2, CXCR4, and SDF-1alpha. J Clin Endocrinol Metab 96: E1388-E1398, 2011.

24. Zhou J, Tian Y, Li J, Lu B, Sun M, Zou Y, Kong R, Luo Y, Shi Y, Wang $\mathrm{K}$ and Ji G: miR-206 is down-regulated in breast cancer and inhibits cell proliferation through the up-regulation of cyclinD2. Biochem Biophys Res Commun 433: 207-212, 2013.

25. Dong Q, Meng P, Wang T, Qin W, Qin W, Wang F, Yuan J, Chen Z, Yang A and Wang H: MicroRNA let-7a inhibits proliferation of human prostate cancer cells in vitro and in vivo by targeting E2F2 and CCND2. PLoS One 5: e10147, 2010. 